\title{
Assessment of a new biological complex efficacy on dysseborrhea, inflammation, and Propionibacterium acnes proliferation
}

This article was published in the following Dove Press journal:

Clinical, Cosmetic and Investigational Dermatology

31 August 2016

Number of times this article has been viewed

\author{
Sandra Trompezinski' \\ Sophie Weber' \\ Benoît Cadars ${ }^{2}$ \\ Florence Larue' \\ Nathalie Ardiet' \\ Marlène \\ Chavagnac-Bonneville ${ }^{2}$ \\ Michèle Sayag ${ }^{2}$ \\ Eric Jourdan ${ }^{2}$
}

'NAOS, Aix-en Provence, ${ }^{2}$ Direction Scientifique Bioderma (NAOS), Lyon, France
Correspondence: Eric Jourdan

Direction Scientifique Bioderma (NAOS),

75 cours Albert Thomas, 69447 Lyon

Cedex 03, France

Tel +33472II 48I6

Email e.jourdan@bioderma.com
Introduction: Acne vulgaris is a common chronic inflammatory disease of the pilosebaceous unit triggered by Propionibacterium acnes. A bakuchiol, Ginkgo biloba extract, and mannitol (BGM) complex has been developed to provide patients with acne with a specific dermocosmetic to be used adjunctively with conventional treatments.

Objective: The aim of these studies was to assess the antibacterial, anti-inflammatory, and antioxidative potential of BGM complex and its individual compounds as well as its impact on sebum composition.

Methods: The antibacterial, anti-inflammatory, and antioxidative potential of BGM complex and its compounds was assessed through in vitro, ex vivo, and clinical studies. The clinical benefit of BGM complex formulated in a cream was assessed in subjects prone to acne through sebum composition analysis and photometric assessments.

Results: Results from the studies showed that the BGM complex has significant antibacterial, anti-inflammatory, and antioxidative properties. At similar concentrations, bakuchiol has up to twice the antioxidative potential than vitamin E. In subjects, BGM complex regulated the sebum composition in acne patients by increasing the level of sapienic and linolenic acid and reducing the level of oleic acid. The reduced number of porphyrins on the skin surface showed that it is also effective against $P$. acnes.

Conclusion: BGM complex provides a complete adjunctive care in patients with acne by targeting etiopathogenic factors of acne: dysseborrhea, inflammation, and P. acnes proliferation.

Keywords: acne, antibacterial, anti-inflammatory, antioxidation, cosmetics, sebum

\section{Introduction}

Acne vulgaris is a common chronic inflammatory disease of the pilosebaceous unit. Its pathophysiology includes abnormal keratinization leading to follicular plugging (comedone formation), inflammation, androgen-mediated stimulation of the sebaceous gland, and proliferation of Propionibacterium acnes within the follicle. ${ }^{1} P$. acnes culture supernatants contain lipases and have been shown to activate inflammatory receptors, such as toll-like receptor $2 .^{2,3}$ More recently, $P$. acnes has been shown to activate proteinase-activated receptor in human keratinocytes when tested in vitro. Proteinase-activated receptor 2 is known for regulating the encoding of several proinflammatory cytokine genes, such as interleukin-1 $\alpha$ (IL-1 $\alpha)$, IL-8, and tumor necrosis factor $\alpha(\mathrm{TNF}-\alpha){ }^{4}$

The hormonal-mediated stimulation of the sebaceous gland observed in patients with acne leads to increased sebum production and potentially changes its composition. ${ }^{5}$ 
Recently named "dysseborrhea" by Melnik, it facilitates both inflammatory and noninflammatory lesion formation. ${ }^{6}$ This change of sebum composition has been specifically observed for squalene, a major sebum component, and linoleic acid. Squalene oxidation is caused by protoporphyrins produced by $P$. acnes and activated by ultraviolet light. Oxidized squalene, which is not present in the sebum of acne-free individuals, has been found in patients with acne. It forms highly comedogenic and sticky polymers, which may lead to hyperkeratinization and inflammation of comedones. ${ }^{7-9}$

To limit the potentially harmful effects of oxidized squalene, the skin defends itself by secreting vitamin $\mathrm{E}$ on its surface. This lipophilic vitamin is present in the cutaneous lipids as a normal component of human sebum. At sites with a high density of sebaceous glands, such as cheeks and the forehead, vitamin $\mathrm{E}$ is continuously secreted at elevated levels, which is correlated with squalene levels. In individuals with acne, vitamin E levels in the sebum are decreased compared with that of healthy individuals, resulting in an increased level of oxidized squalene. ${ }^{10-12}$

Linoleic acid, another compound of the human sebum, is an essential fatty acid. It participates in the preservation of the skin barrier integrity and a composant of stratum corneum ceramides. In acne patients, the quantity of linoleic acid in epidermal lipids and comedones decreases with increasing severity. This decrease in linoleic acid leads to the impaired skin barrier function and predisposes the skin to infections. ${ }^{13}$ In addition, linoleic acid inhibits $5 \alpha$-reductase activity, which regulates sebum production. ${ }^{14}$ In the skin, linoleic acid is not metabolized into $\gamma$-linolenic acid but is primarily transformed into 13-hydroxyoctadecadienoic acid, which has antiproliferative and anti-inflammatory properties. ${ }^{15}$ When applied on the skin, linoleic acid reduces the size of comedones by significantly reducing their growth. ${ }^{16}$

Oleic acid also belongs to the essential fatty acid group and is part of the hydrolipidic film. Applying oleic acid on skin causes comedones as it impairs the skin barrier permeability through changes in the membrane structure of the stratum corneum and decreases the epidermal calcium gradient. ${ }^{17,18}$ It acts on skin via receptors in the calcium channels, increasing the influx of calcium into the keratinocytes, thereby causing epidermal hyperplasia, poor keratinization, and scaly skin. In addition, oleic acid has been shown to cause $P$. acnes proliferation and localized inflammation by increasing the production of IL- $1 \alpha$ by keratinocytes. ${ }^{7}$

Sapienic acid has antibacterial properties and is the most abundant fatty acid released from triglycerides in sebum. ${ }^{10}$
The present article reports on results from in vitro, ex vivo, and clinical studies conducted with a new dermocosmetic (Sebium $^{\circledR}$ Global, Laboratoire Bioderma [NAOS], France) or its ingredients (bakuchiol, Ginkgo biloba extract, and mannitol [BGM] complex) assessing its antibacterial, antiinflammatory, sebum protection, and antioxidation potential in the regulation process of the physiopathology of acne.

BGM complex is composed of natural compounds: the antibacterial and anti-inflammatory compound bakuchiol, anti-inflammatory Ginkgo biloba extract, and antioxidant mannitol. ${ }^{19-23}$

In the past, individual compounds of BGM complex have demonstrated their potential benefits in reducing infections, inflammation, and oxidation. ${ }^{19,20,22,24,25}$ The BGM complex was formulated and developed to offer individuals prone to acne with a moisturizing cream, which in addition to managing dryness, provides an adjunctive benefit to therapeutics as demonstrated recently in a clinical study. ${ }^{26}$

\section{Methods}

\section{Antibacterial activity}

\section{In vitro testing}

The antibacterial activity of BGM complex against $P$. acnes (strain CIP A 179) was assessed in vitro through the determination of the minimum inhibitory concentration (MIC) using standard methods. P. acnes was cultivated in a WilkinsChalgren liquid culture medium at $37^{\circ} \mathrm{C} \pm 1^{\circ} \mathrm{C}$ for 24 hours. A tryptone salt solution was prepared to obtain a bacterial concentration of $10^{7} \mathrm{CFU} / \mathrm{mL}$ measured using a densitometer (optical density $=1$, Densimat ${ }^{\circledR}$, bioMérieux, Marcy-l'Etoile, France). This suspension was diluted ten times to obtain a final dilution of $10^{-6}$ allowing for an accurate cell count.

The MIC of BGM complex actives was tested compared to zinc gluconate, benzoyl peroxide (BPO), and the antibiotic erythromycin. Sterilized and osmosed water was used as a solvent for Gingko biloba extract, mannitol, and zinc gluconate. Dimethyl sulfoxide (DMSO, an organic solvent), acetone, and methanol were used as a solvent for bakuchiol, BPO, and erythromycin, respectively. These solvents also served as negative control. The compounds were tested at up to nine concentrations. Each solution $(2 \mathrm{~mL})$ was placed in a Petri dish and covered with Wilkins-Chalgren agar medium $(18 \mathrm{~mL})$. After solidification, each dish was inoculated with a calibrated suspension of the $P$. acnes strain to be tested. Cultures were incubated under anaerobic conditions with the test products for 48 hours at $37^{\circ} \mathrm{C}$. The MIC corresponded to the lowest concentration of the active for which no visible culture was observed (up to three colonies were considered not relevant). 


\section{Clinical testing}

The antibacterial activity of a topical cream containing BGM complex and other compounds, including zinc gluconate, was tested using VISIA CAS (Parsippany, NJ, USA) numeric photographs. Assessments were made on porphyrins visible under ultraviolet light on the face as white spots. Assessments were made in 17 healthy volunteers aged 19 to 34 years with both oily skin and mild to moderate facial acne (minimum five inflammatory lesions) having provided written informed consent. Participants applied BGM complex in a cream formulation twice daily for 84 days. No product was to be applied at visit days 28, 56, and 84. Prior to each examination, participants cleansed their face using a cotton swab with $2 \mathrm{~mL}$ (Crealine ${ }^{\circledR}$ Sensibio $^{\circledR}$, Laboratoire Bioderma [NAOS], France).

The percentage of the mean variation value was calculated for each parameter using the following formula:

$$
\Delta \%=\left[\left(\mathrm{T}_{\mathrm{ti}}-\mathrm{T}_{\mathrm{t} 0}\right) / \mathrm{T}_{\mathrm{t} 0}\right] \times 100
$$

with $\mathrm{T}_{\mathrm{ti}}=$ measured on the treated zone at time $\mathrm{t}_{\mathrm{i}}$ and $\mathrm{T}_{\mathrm{t} 0}$ $=$ measured on the treated zone at time $t_{0}$.

The Student's $t$-test was used to compare variations at the different study visits compared to day 0 and between compounds.

\section{In vitro testing of the antioxidation activity of squalene}

The antioxidation potential of three concentrations $(3.9,9.5$, and $19 \mathrm{mM}$ ) of bakuchiol was tested in vitro compared to two vitamin E samples at 3.9 and $19 \mathrm{mM}$. Hydrogen peroxide at $15 \%$ was used as an oxidation agent. Hydrogen peroxide and the antioxidation agents were added to squalene. Stigmasterol $25 \mathrm{mg}$ in $5 \mathrm{~mL}$ of chloroform was used as an internal standard and added to both bakuchiol and vitamin E-treated samples.

Squalene was quantified after incubation at room temperature for 1 hour and extraction using gas chromatography coupled with mass spectrometry: an Agilent 6890N (Agilent Technologies, Santa Clara, CA, USA) gas chromatography unit equipped with an on-column injector was coupled to a Agilent 5975 Mass Selective Detector mass spectrometer via a high-temperature interface. Separation was achieved using a $30 \mathrm{~m} \times 0.25 \mathrm{~mm} \times 0.1 \mu \mathrm{m}$ ZB-1HT capillary column (Phenomenex $^{\circledR}$, Torrance, CA, USA). Helium was used as a carrier gas at a constant flow of $0.8 \mathrm{~mL} / \mathrm{min}$. Injector and transfer line temperatures were set to $250^{\circ} \mathrm{C}$ and $300^{\circ} \mathrm{C}$, respectively. EI mass spectra were recorded in a total ion current monitoring mode. EI mass spectra operating conditions were source temperature at $250^{\circ} \mathrm{C}$, ionizing energy at $70 \mathrm{eV}$, and a scan range from 100 to $500 \mathrm{~m} / \mathrm{z}$.
The Kruskal-Wallis test was used to compare the distribution of lipid variables.

\section{Ex vivo testing of the anti-inflammatory activity}

A total of 15 human skin explants with a diameter of $10 \pm 1$ $\mathrm{mm}$ were obtained from the abdomen of a 40 -year-old female subject who underwent plastic surgery and had provided written donor consent. Explants were cultured in a Bio EC's explant medium at $37^{\circ} \mathrm{C}$ in a humid atmosphere enriched with $5 \%$ of $\mathrm{CO}_{2}$. A cream containing BGM complex was applied twice daily for 3 days (days 0,1 , and 2) at a dose of $2 \mathrm{mg} / \mathrm{cm}^{2}$; one sample group did not receive any product application. On days 1 and 2, a paper disk containing $30 \mu \mathrm{L}$ of a reconstituted lyophilized $P$. acnes strain was applied for 1 hour, twice daily, on the surfaces of all explants, except for the control $(\mathrm{n}=5)$. Skin explants not exposed to $P$. acnes or BGM complex served as control samples. For samples receiving the test product, this was made prior to product application. On day 3, culture medium samples were taken and frozen at $-80^{\circ} \mathrm{C}$.

The concentration of IL- 8 and TNF- $\alpha$ in the culture medium samples was assessed using an ELISA kit according to provider's instructions (IL-8: Cat\# EH-IL8-001, RayBio $^{\circledR}$, Norcross, GA, USA; TNF- $\alpha$ : 589201, Cayman Inc., Ann Arbor, MI, USA). Absorption was assessed using the multimode reader Tecan Infinite M200Pro, Tecan Inc., Männedorf, Switzerland. IL-8 quantification was expressed in $\mathrm{ng} / \mathrm{mL}$ and $\mathrm{TNF}-\alpha$ in $\mathrm{pg} / \mathrm{mL}$.

The Student's $t$-test was used for statistical purposes. The probability level was set at 5\%.

\section{Assessment of the sebum composition}

A study conducted between February and April 2011 assessed the quantitative and qualitative sebum composition in 38 healthy volunteers with acne prone skin and ten healthy volunteers with no acne. Prior to any inclusion, the study received authorization from the relevant ethics committee of the investigational site at Villeurbanne, France and French health regulations under the follwing number: 10E3092. Subjects were all Caucasians, had predominantly a skin phototype III, and were aged between 18 and 40 years.

Subjects were randomized on site to receive for 56 days either the placebo cream or BGM complex in a cream.

Sebum from the forehead was sampled on days 0,28 , and 56 using genuine glassware Sebum kit (Synelvia, Labège, France). Lipids were eluted in inert organic solvent and frozen at $-20^{\circ} \mathrm{C}$ prior to analyses. 
The analysis of the sebum composition through specific sebum markers comprising nonoxidized squalene, oleic acid, linoleic acid, and sapienic acid was performed using the gas chromatography coupled with mass spectrometry method as detailed earlier.

The Student's $t$-test was used for statistical purposes. The probability level was set at $5 \%$.

\section{Results}

\section{Antibacterial activity}

In vitro testing

Results from this in vitro study showed that the MIC inhibiting P. acnes growth was $0.0005 \%$ for bakuchiol, $0.00002 \%$ for mannitol, and $0.0002 \%$ for Ginkgo biloba extract. MIC for zinc gluconate was $0.12 \%, 0.008 \%$ for BPO, and $0.0000125 \%$ for erythromycin. DMSO, sterilized and osmosed water, acetone, and methanol had no antibacterial activity against $P$. acnes at concentrations used to solubilize tested compounds.

\section{Clinical testing}

Results from the clinical assessments of the porphyrins parameter on the face (average scoring) showed a significant decrease $(P<0.05)$ as early as day $28(0.0144 \pm 0.0032)$ from day $0(0.0203 \pm 0.0042)$, which was sustained until day 56 $(0.0128 \pm 0.002905)$. Due to an increase of porphyrins in three participants, the difference was not statistically significant at day $84(0.0154 \pm 0.0034)$ for the front of the face. When evaluating the right and left sides of the face, a statistically significant decrease at all post-day 0 visits was observed $(P<0.05)$ (Figure 1).

\section{In vitro testing of the antioxidative activity of squalene}

The antioxidative potential of bakuchiol was assessed in a model using squalene previously oxidized by $\mathrm{H}_{2} \mathrm{O}_{2}$. Bakuchiol protected squalene from oxidation by $30.0 \%$ at a concentration of $3.9 \mathrm{mM}$ and $36.9 \%$ at $19 \mathrm{mM}$ compared to $15.2 \%$ and $40.3 \%$ for vitamin $\mathrm{E}$ at the same concentrations (Figure 2).

Under the study conditions, the squalene protection index of Bakuchiol was as twice as high as that of vitamin $\mathrm{E}$.

\section{Ex vivo testing of the anti-inflammatory activity \\ IL-8}

For untreated explants, the IL-8 level in supernatants was $59.3 \pm 8.1 \mathrm{ng} / \mathrm{mL}$ after 3 days. Following 3 days incubation of skin samples with $P$. acnes, IL-8 secretion increased by $92 \%$ $(114.0 \pm 9.4 \mathrm{ng} / \mathrm{mL})$. Applying BGM complex cream resulted in a significant limited increase of $45 \%(89.6 \pm 4.6 \mathrm{ng} / \mathrm{mL}$, $P<0.01)$ of $P$. acnes-induced IL-8 secretion (Figure $3 \mathrm{~A}$ ).

\section{TNF- $\alpha$}

For untreated skin explants, the TNF- $\alpha$ level in supernatants was $9.9 \pm 1.8 \mathrm{pg} / \mathrm{mL}$ after 3 days. Incubation for 3 days with

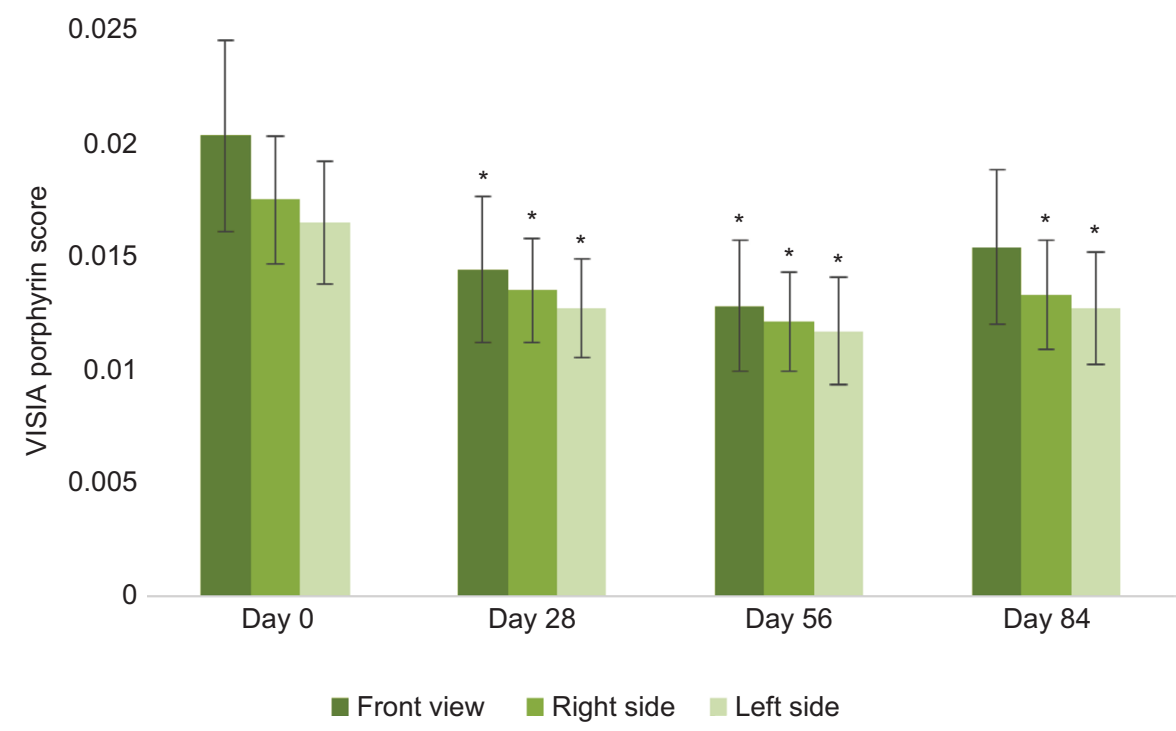

Figure I Decrease of porphyrin score over time after application of BGM complex twice daily.

Note: ${ }^{* P}<0.05$ compared to day 0 ; porphyrins were assessed at days $0,28,56$, and 84 using ultraviolet light and the VISIA CAS numeric photographic system (Canfield, UK) after twice daily application of BGM complex.

Abbreviation: BGM, bakuchiol, Ginkgo biloba extract, and mannitol. 


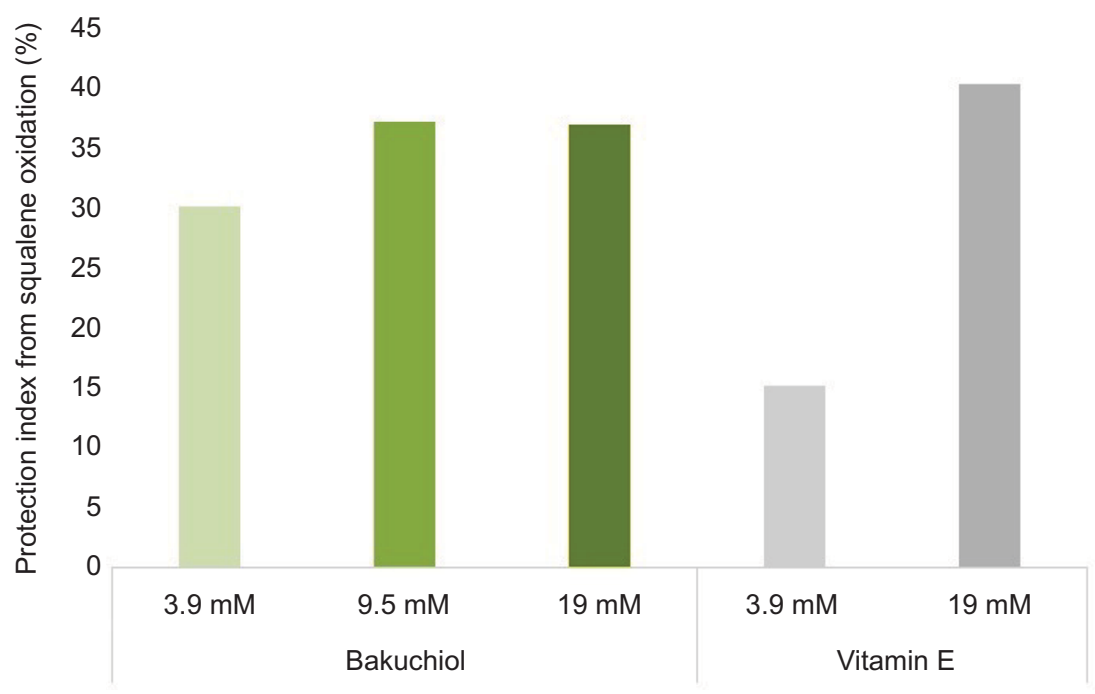

Figure 2 The antioxidative potential of bakuchiol compared to vitamin E, tested in vitro.

$P$. acnes caused an increase of TNF- $\alpha$ secretion of $138 \%$ $(23.6 \pm 3.1 \mathrm{pg} / \mathrm{mL})$. Applying a BGM complex cream resulted in a significant limited increase of $46.0 \%(17.3 \pm 2.0 \mathrm{pg} / \mathrm{mL}$, $P<0.01)$ of $P$. acnes-induced TNF- $\alpha$ secretion (Figure $3 \mathrm{~B}$ ).

\section{Assessment of the sebum composition}

Regular applications of BGM complex in a cream over 56 days improved the assessed sebum markers in subjects prone to acne: the quantity of nonoxidized squalene increased by $27 \%, 37 \%$ for linoleic acid, and $10 \%$ for sapienic acid; for oleic acid, it had decreased by $10 \%$ (Figure 4). Statistically significant differences compared to day 0 were observed after 56 days $(P<0.05)$. No remarkable changes from day 0 were observed in the placebo group. After 56 days of application, the sebum composition produced by subjects with acne was similar to that of subjects without acne.

\section{Discussion}

Acne is a multifactorial condition with a complex pathophysiology. Management currently combines pharmacologically active substances, such as topical retinoids, antibiotics, or other molecules alone or combined with each other, as well as dermocosmetics to prevent dryness and erythema caused by such medications. ${ }^{27}$

The present work reports in vitro, ex vivo, and clinical data about BGM complex, a new dermocosmetic compound for the adjunctive management of acne.

Under in vitro test conditions, the BGM complex has antibacterial properties against $P$. acnes similar to other currently known antibacterial agents, such as zinc gluconate, $\mathrm{BPO}$, and erythromycin. ${ }^{21,28-31}$ Clinical testing of the BGM complex cream using a photometric quantification method through ultraviolet light fluorescence of porphyrins generated by $P$. acnes confirmed the in vitro results: BGM complex significantly reduces the number of porphyrins by inhibiting P. acnes growth as early as 28 days after the first application and at day 56. The fact that its efficacy on the front side of the face was not significantly sustained until day 84 may be due to the small sample size and the fact that three subjects had an increased porphyrin count at the end of the study, biasing the present results.

Ex vivo testing of its anti-inflammatory potential showed a significant decreased synthesis of IL- 8 and TNF- $\alpha$ cytokines caused by $P$. acnes, suggesting a reduced inflammation associated with acne lesions.

In vitro testing of the antioxidation potential confirmed that at a molecular concentration of $3.9 \mathrm{mM}$, bakuchiol was twice as effective as vitamin $\mathrm{E}$ in reducing squalene oxidation. This result indicates that bakuchiol may allow the temporary replacement of naturally produced vitamin $\mathrm{E}$ in individuals with acne, thus re-equilibrating the qualitative balance of sebum.

We confirmed these results through a clinical study. After 56 days of treatment, the lipid profile analyzed by gas chromatography coupled with mass spectrometry demonstrated that the rate of nonoxidized squalene had increased by $17 \%$ compared to day 0 and by $27 \%$ when compared to placebo while acne-prone subjects presented with a sebum lipid composition very similar to that of healthy subjects: the decreased levels of sapienic and linoleic acid and the increased level of oleic acid observed in subjects with acne returned to levels observed in normal skin.

The present study results complete those shown through a recently reported clinical study in 111 patients with acne who received a retinoid (adapalene) and BGM complex in 
A

Exposed to $P$. acnes

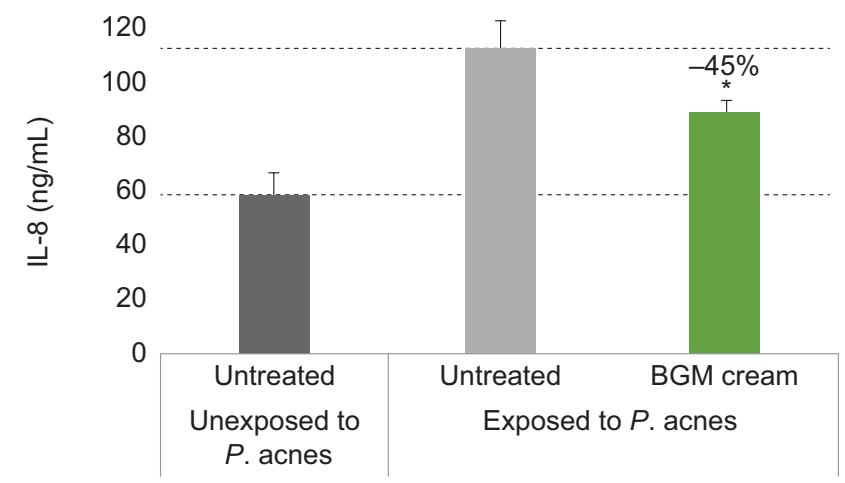

B

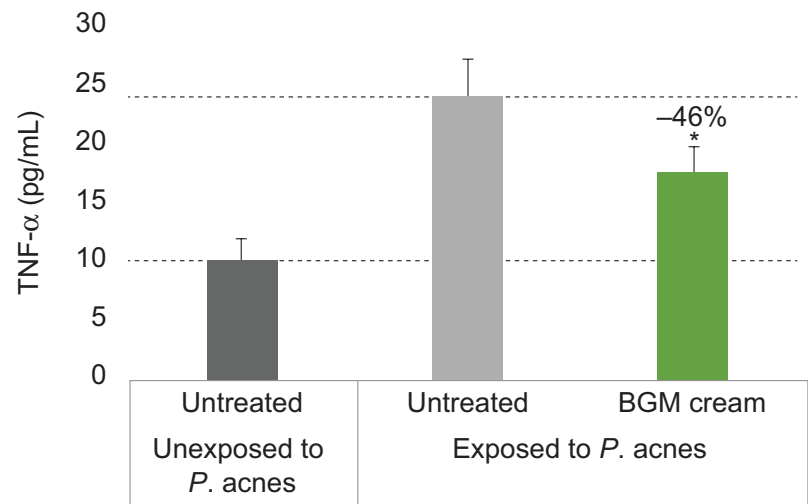

Figure 3 Inhibition of $P$. acnes-induced inflammatory cytokines.

Notes: Mean concentration of (A) IL-8 and (B) TNF- $\alpha$ in supernatants after 3 days of application of a neutral formulation with BGM complex compared to unexposed and untreated skin explants. Differences were significant in favor $(P<0.0 \mathrm{I})$ of $B G M$ complex in a cream for samples exposed to $P$. acnes, $* P<0.01$.

Abbreviations: BGM, bakuchiol, Ginkgo biloba extract, and mannitol; IL-8, interleukin 8; P. acnes, Propionibacterium acnes; TNF- $\alpha$, tumor necrosis factor $\alpha$.

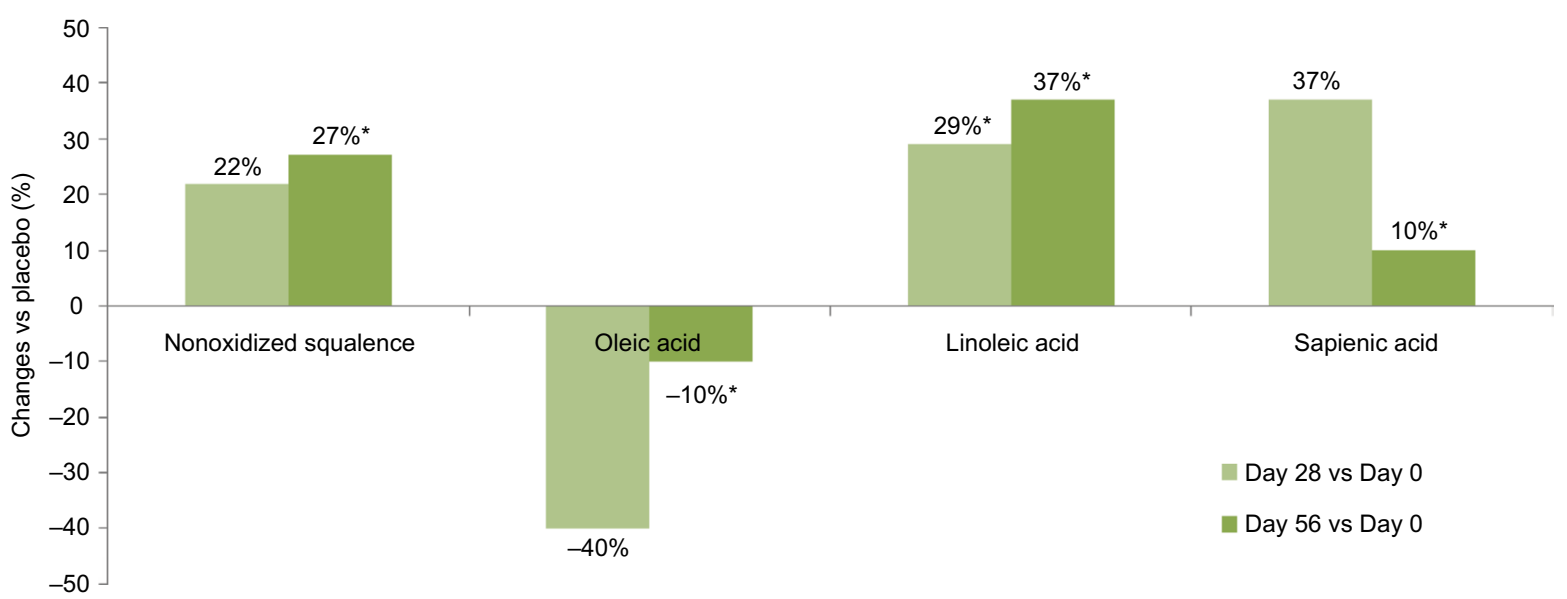

Figure 4 Sebum composition: percent changes from day 0 at days 28 and 56 with BGM complex in patients with acne.

Note: $* P<0.05$ versus day 0 ; quantitative and qualitative sebum composition was assessed at days 0,28 , and 56 using GC/MS.

Abbreviations: BGM, bakuchiol, Ginkgo biloba extract, and mannitol; GC/MS, gas chromatography coupled with mass spectrometry.

a cream or a placebo cream. This study demonstrated that the BGM complex improves the treatment outcome of the retinoid in patients with acne vulgaris. ${ }^{26}$

\section{Conclusion}

The BGM complex acts directly on acne in reducing $P$. acnes and inflammation, inhibiting squalene oxidation and restoring healthy levels of fatty acids in the sebum.

By having an impact on the major parameters involved in acne, the BGM complex may provide a complete adjunctive care in patients with acne.

\section{Acknowledgments}

The authors acknowledge the writing support of Patrick Göritz, SMWS-Scientific and Medical Writing Services, France. The studies were funded by Bioderma (NAOS), Lyon.

\section{Disclosure}

The authors are employees of NAOS, Aix-en-Provence, France or of Bioderma (NAOS), Lyon, France. The authors report no other conflicts of interest in this work.

\section{References}

1. Haider A, Shaw JC. Treatment of acne vulgaris. JAMA. 2004; 292(6):726-735.

2. Hoeffler U. Enzymatic and hemolytic properties of Propionibacterium acnes and related bacteria. J Clin Microbiol. 1977;6(6):555-558.

3. Jugeau S, Tenaud I, Knol AC, Jarrousse V, Quereux G, Khammari A, Dreno B. Induction of toll-like receptors by Propionibacterium acnes. Br J Dermatol. 2005;153(6):1105-1113.

4. Komatsu H, Enjouji S, Ito A, Ohama T, Sato K. Prostaglandin E(2) inhibits proteinase-activated receptor 2-signal transduction through regulation of receptor internalization. J Vet Med Sci. 2013;75(3):255-261.

5. Saint-Leger D, Bague A, Lefebvre E, Cohen E, Chivot M. A possible role for squalene in the pathogenesis of acne. II. In vivo study of squalene oxides in skin surface and intra-comedonal lipids of acne patients. $\mathrm{Br}$ J Dermatol. 1986;114(5):543-552. 
6. Melnik BC. Linking diet to acne metabolomics, inflammation, and comedogenesis: an update. Clin Cosmet Investig Dermatol. 2015;8: 371-388.

7. Chiba K, Yoshizawa K, Makino I, Kawakami K, Onoue M. Comedogenicity of squalene monohydroperoxide in the skin after topical application. J Toxicol Sci. 2000;25(2):77-83.

8. Ottaviani M, Alestas T, Flori E, Mastrofrancesco A, Zouboulis CC, Picardo M. Peroxidated squalene induces the production of inflammatory mediators in $\mathrm{HaCaT}$ keratinocytes: a possible role in acne vulgaris. J Invest Dermatol. 2006;126(11):2430-2437.

9. Tochio T, Tanaka H, Nakata S, Ikeno H. Accumulation of lipid peroxide in the content of comedones may be involved in the progression of comedogenesis and inflammatory changes in comedones. $J$ Cosmet Dermatol. 2009;8(2):152-158.

10. Drake DR, Brogden KA, Dawson DV, Wertz PW. Thematic review series: skin lipids. Antimicrobial lipids at the skin surface. J Lipid Res. 2008;49(1):4-11.

11. Picardo M, Ottaviani M, Camera E, Mastrofrancesco A. Sebaceous gland lipids. Dermatoendocrinol. 2009;1(2):68-71.

12. Thiele JJ, Weber SU, Packer L. Sebaceous gland secretion is a major physiologic route of vitamin E delivery to skin. $J$ Invest Dermatol. 1999;113(6):1006-1010

13. Ottaviani M, Camera E, Picardo M. Lipid mediators in acne. Mediators Inflamm. 2010;2010. pii:858176.

14. Namazi MR. Further insight into the pathomechanism of acne by considering the 5-alpha-reductase inhibitory effect of linoleic acid. Int J Dermatol. 2004;43(9):701.

15. Ziboh VA, Miller CC, Cho Y. Metabolism of polyunsaturated fatty acids by skin epidermal enzymes: generation of antiinflammatory and antiproliferative metabolites. Am J Clin Nutr. 2000;71(1 Suppl):361S-366S.

16. Letawe C, Boone M, Pierard GE. Digital image analysis of the effect of topically applied linoleic acid on acne microcomedones. Clin Exp Dermatol. 1998;23(2):56-58.

17. Choi EH, Ahn SK, Lee SH. The changes of stratum corneum interstices and calcium distribution of follicular epithelium of experimentally induced comedones (EIC) by oleic acid. Exp Dermatol. 1997;6(1):29-35.

18. Katsuta Y, Iida T, Hasegawa K, Inomata S, Denda M. Function of oleic acid on epidermal barrier and calcium influx into keratinocytes is associated with $\mathrm{N}$-methyl D-aspartate-type glutamate receptors. $\mathrm{Br}$ J Dermatol. 2009;160(1):69-74.

19. Kaul R. [Kinetics of the anti-staphylococcal activity of bakuchiol in vitro (author's transl)]. Arzneimittelforschung. 1976;26(4):486-489.
20. Katsura H, Tsukiyama RI, Suzuki A, Kobayashi M. In vitro antimicrobial activities of bakuchiol against oral microorganisms. Antimicrob Agents Chemother. 2001;45(11):3009-3013.

21. Mohammad TF, Burkhart CG. Acne therapeutics: a closer look at benzoyl peroxide. Skinmed. 2015;13(2):94-96.

22. Ferrandiz ML, Gil B, Sanz MJ, et al. Effect of bakuchiol on leukocyte functions and some inflammatory responses in mice. J Pharm Pharmacol. 1996;48(9):975-980.

23. Pelle E, Huang X, Mammone T, Marenus K, Maes D, Frenkel K. Ultraviolet-B-induced oxidative DNA base damage in primary normal human epidermal keratinocytes and inhibition by a hydroxyl radical scavenger. J Invest Dermatol. 2003;121(1):177-183.

24. Feng X, Zhang L, Zhu H. Comparative anticancer and antioxidant activities of different ingredients of Ginkgo biloba extract (EGb 761). Planta Med. 2009;75(8):792-796.

25. Liu JH, Chen MM, Huang JW, et al. Therapeutic effects and mechanisms of action of mannitol during $\mathrm{H}_{2} \mathrm{O}_{2}$-induced oxidative stress in human retinal pigment epithelium cells. J Ocul Pharmacol Ther. 2010;26(3): 249-257.

26. Polakova K, Fauger A, Sayag M, Jourdan E. A dermocosmetic containing bakuchiol, Ginkgo biloba extract and mannitol improves the efficacy of adapalene in patients with acne vulgaris: result from a controlled randomized trial. Clin Cosmet Investig Dermatol. 2015;8:187-191.

27. Goreshi R, Samrao A, Ehst BD. A double-blind, randomized, bilateral comparison of skin irritancy following application of the combination acne products clindamycin/tretinoin and benzoyl peroxide/adapalene. J Drugs Dermatol. 2012;11(12):1422-1426.

28. Faghihi G, Isfahani AK, Hosseini SM, Radan MR. Efficacy of intense pulsed light combined with topical erythromycin solution $2 \%$ versus topical erythromycin solution $2 \%$ alone in the treatment of persistent facial erythematous acne macules. Adv Biomed Res. 2012;1:70.

29. Dutil M. Benzoyl peroxide: enhancing antibiotic efficacy in acne management. Skin Therapy Lett. 2010;15(10):5-7.

30. Jasson F, Nagy I, Knol AC, Zuliani T, Khammari A, Dreno B. Different strains of Propionibacterium acnes modulate differently the cutaneous innate immunity. Exp Dermatol. 2013;22(9): 587-592.

31. Bhatia N, Pillai R. Randomized, observer-blind, split-face compatibility study with clindamycin phosphate $1.2 \%$ /benzoyl peroxide $3.75 \%$ gel and facial foundation makeup. J Clin Aesthet Dermatol. 2015;8(9):25-32.
Clinical, Cosmetic and Investigational Dermatology

\section{Publish your work in this journal}

Clinical, Cosmetic and Investigational Dermatology is an international, peer-reviewed, open access, online journal that focuses on the latest clinical and experimental research in all aspects of skin disease and cosmetic interventions. This journal is included on PubMed. The manuscript management system is completely online
Dovepress

and includes a very quick and fair peer-review system, which is all easy to use. Visit http://www.dovepress.com/testimonials.php to read real quotes from published authors 